\title{
Regeneration of Lesioned CNS Axons by Neutralisation of Neurite Growth Inhibitors: A Short Review
}

\author{
M. E. Schwab \\ Brain Research Institute, University of Zurich, August-Forel-Str. 1, CH-8029 Zurich, \\ Switzerland
}

Key words: Regeneration; Neurite growth inhibitor; Myelin; Oligodendrocyte; CNS lesion

Traumatic lesions of the nervous system induce a variety of changes: degeneration of the distal axon stump, in many peripheral and central pathways followed by sprouting of the proximal stump, reactions in the surrounding glia, and immigration of macrophages. A fundamental difference between peripheral and central nervous system appears thereafter: in peripheral nerves regenerative sprouts often elongate and regrow over long distances and may finally reestablish functional connections. In contrast, regenerative fibre growth does not occur in the central nervous system (CNS). ${ }^{1}$ Rather, arrest of growth, retraction of the fibres, and formation of retraction bulbs is observed. The biological reasons for this different behaviour were very unclear until recently. In animal experiments transplantations of pieces of peripheral nerves into various regions of the brain and spinal cord showed the crucial role of the local tissue environment. ${ }^{2-4}$ In these experiments many different types of CNS neurons successfully regenerated their lesioned axons in a peripheral nerve bridge. However, axon growth subsided as soon as these regenerating fibres contacted CNS tissue. ${ }^{2}$ Classically, these results were interpreted as a lack of growth stimulating neurotrophic factors in CNS tissue. Tissue and cell culture experiments in which we studied the interaction of growing neurites with various parts of the CNS and with different CNS glial cell types suggested an alternative explanation: the presence of inhibitors of neurite growth. ${ }^{5-7}$

\section{CNS myelin contains inhibitors of neurite growth}

When dissociated neurons of perinatal rats were plated on frozen sections of adult rat spinal cord, cerebellum or forebrain, neurites grew exclusively on the grey matter areas and strongly avoided white matter regions. ${ }^{6}$ Similarly, neurites never 
grew into adult rat optic nerve explants in spite of a strong stimulation of fiber growth by neurotrophic factors (NGF, BDNF). ${ }^{7}$ The analysis on the single cell level demonstrated a very different effect of the two main classes of CNS glial cells on growing neurites: whereas cultured astrocytes were permissive or favourable substrates for neurite growth, contact of neurite growth cones with oligodendrocytes lead to a rapid and long-lasting paralysis of the growing fibres. ${ }^{8,9}$ Growth inhibition was also exerted by isolated CNS (but not PNS) myelin and by extracted myelin proteins. Extensive biochemical characterisation lead to the identification of two so far unknown myelin proteins with the molecular weight of 35000 and $250000 \mathrm{Da} .{ }^{10}$ These proteins exert a powerful and long-lasting inhibitory effect on neurite growth and were therefore called neurite growth inhibitors NI-35 and NI250. Biochemically and immunologically they are closely related; cDNA cloning and structural analysis is currently being done. Monoclonal antibodies neutralising their inhibitory activity were produced and used to determine the role of these neurite growth inhibitors in the lesioned CNS. ${ }^{11}$ Studies on the occurrence and distribution of NI-35/250 showed that they selectively occur in oligodendrocytes and CNS myelin as tightly linked membrane proteins, and they were found in species like the rat, cow, and chick, and probably occur in all higher vertebrates. In contrast, they are absent from peripheral nerves, other tissues and organs, and they were not found in the CNS of fish. (Fish are known to regenerate lesioned CNS pathways after lesion.)

\section{Inactivation of neurite growth inhibitors in the lesioned adult CNS: effects on} axon regeneration

Two different, independent methods were used to eliminate or neutralise these neurite growth inhibitors. In the first experimental paradigm newborn rats were locally treated with a high dose of $\mathrm{X}$-rays, a procedure which has been shown to permanently eliminate oligodendrocytes, thus producing myelin-free CNS tissue. ${ }^{12,13}$ The second paradigm consisted of the application of the monoclonal antibody IN-1 which neutralises the inhibitory activity of NI-35 and NI-250. ${ }^{14}$ Interestingly, the results obtained in the lesioned spinal cord and optic nerve with both procedures were very similar: axons regenerating over long distances were observed under these conditions.

Two to seven week old rats were lesioned at T5 by a bilateral transection of the dorsal two thirds of the spinal cord, leaving only the ventral funiculi intact. This lesion completely interrupts the corticospinal tract (CST) on both sides. (The CST runs in the medial part of the dorsal funiculi in the rat, and the ventral uncrossed component consists only of very few axons.) After survival times of 2 to 6 weeks the CST was labelled by anterograde transport of the lectin-marker complex wheat germ agglutinin-horseradish peroxidase (WGA-HRP). Consistently, spontaneous sprouting of the lesioned CST fibres was observed at the lesion site, and some of these sprouts were able to reach the caudal side of the lesion. Elongation was measured from the caudal end of the lesion and amounted to 0.1 to $1 \mathrm{~mm}$ in the control groups (untreated, normally myelinated rats, or rats treated with control antibodies). ${ }^{1314}$ Very much in contrast, rats with myelin-free spinal cords (due to neonatal X-irradiation), ${ }^{13}$ or myelinated rats treated with the inhibitor neutralising antibody $\mathrm{IN}-1^{14}$ showed elongation distances of 2.5 to $18.7 \mathrm{~mm}$. In contrast to the 
very straight course of normal CST fibres, regenerating fibers often followed an irregular course and were frequently seen to branch in the grey matter. Many of these fibres presumably reached former target areas. In spite of the impressive distances of regeneration the number of successfully regrowing fibres was small in all these experiments. A major reason may be the lesion itself which may represent a massive barrier. Indeed, regenerating fibres usually grew around the lesion through the remaining spinal cord tissue rather than through the lesion site itself.

Two other well characterised CNS pathways were investigated for their regeneration capability in response to the inactivation of neurite growth inhibitors: the septo-hippocampal pathway, and the optic nerve. The cholinergic innervation of the dorsal hippocampus orginates in the medial septal nuclei and reaches the hippocampus via the fimbria-fornix. The pathway was interrupted by an aspiration lesion of the fimbria-fornix at the entry into the hippocampus. ${ }^{15}$ The large lesion was then bridged by an implant of extracellular matrix, soaked with NGF to stimulate survival and regrowth of the lesioned cholinergic fibres. Three to five weeks later AchE-positive fibres had crossed the bridge and entered the hippocampus. The distance of elongation was measured from the entry point into the hippocampus. In animals receiving control antibodies fibres had regenerated for 0.1 to 1 $\mathrm{mm}$, a result which is in agreement with several reports in the literature using different types of bridges. ${ }^{16,17}$ In IN-1 antibody treated rats, however, fibres extented over 1.5 to more than $3.5 \mathrm{~mm}$ in the caudal and the lateral direction. ${ }^{15}$ The longest fibres extended throughout the whole extent of the dorsal hippocampus.

Optic nerves were lesioned intracranially by repeated freezing, a procedure which interrupts $100 \%$ of the optic fibers. Spontaneous regeneration in control animals ranged around 0.3 to $0.5 \mathrm{~mm}$. In X-irradiated, myelin- and inhibitor-free optic nerves regenerating axons could be followed-up to the chiasm $(2.5-3.5 \mathrm{~mm})$ (Cadelli, Schnell and Schwab, unpublished observations). A significant enhancement of regeneration was also observed in IN-1 antibody treated rats.

All these experiments show that, consistent with the wide spread occurrence of neurite growth inhibitors in central myelin, regeneration of axons can be observed in very different parts of the CNS following the inactivation of NI-35/250. These inhibitors therefore seem to play a crucial role for the lack of spontaneous regeneration of CNS fibre tracts.

\section{Additional factors influencing CNS lesions and neurite regeneration}

As documented by a vast literature and obvious in all histological examinations of lesion sites the local tissue reactions and the scar formation can represent a significant barrier for regenerating axons. Scar formation greatly depends on the type and extent of the lesion, and includes reactive astrocytes, immigrating fibroblasts, and extracelullar matrix structures like basement membrane and collagen. Experimental work until now has not definitely shown whether these scars hinder regenerating axons only mechanically, or whether additional repulsive or inhibitory factors are expressed. The very active research in this field at present will help to clarify this situation in the near future.

In the normal CNS, in particular during development, many types of neurons depend on specific neurotrophic factors for survival, differentiation, and neurite outgrowth. ${ }^{18}$ In the lesioned adult nervous system neurotrophic factors can prevent 
the atrophy of lesioned neurons and stimulate regeneration. ${ }^{19-20}$ A combined application of neurotrophic factors and antibodies neutralising neurite growth inhibitors can be expected to increase the number of successfully regenerating fibres. Such experiments are currently on the way in our laboratory.

\section{Future outlook}

Several important aspects with regard to neurite growth inhibitors are currently being tackled. Of particular importance is the elucidation of the structure of IN-35 and NI-250, the interaction with presumable receptors on neuronal membranes, and the cell biological mechanism of growth inhibition. We hope that this information will provide us with new clues on how to inactivate or overcome the activity of these molecules during regeneration in order to develop a treatment which ultimately could be transfered to human patients. With the help of modern molecular biological techniques such a goal may not be outside reach.

A second important area of research concerns the formation of connections and the functional consequences of the regenerated fibres. Experiments in fish and recent results using sciatic nerve bridges in the optic system of the rat suggest that regenerating fibres can recognise the cues for specific target interactions and can reform functional connections. ${ }^{4}$ Often, the stabilisation and refinement of these connections seems to be influenced by activity, a process which on the human level would require specific rehabilitation programmes to train and stabilise the recovery of connections and functions.

\section{Acknowledgement}

Our work is supported by grants from the Swiss National Science Foundation, the International Spinal Research Trust, the American Paralysis Association, Springfield/NJ, the Swiss Multiple Sclerosis Society, Regeneron Pharmaceuticals Inc., New York, and the Dr Eric Slack-Gyr-Foundation.

\section{References}

1 Ramon y Cajal S. Degeneration and Regeneration of the Nervous System. 1928 (Engl. transl. and reprint, 1959), Hafner, New York.

2 David S, Aguayo AJ. Axonal elongation into peripheral nervous system 'bridges' after central nervous system injury in adult rats. Science 1981: 214:931-933.

3 Richardson PM, Issa VMK, Aguayo AJ. Regeneration of long spinal axons in the rat. J Neurocytol 1984: 13:165-182.

4 Keirstead SA, Rasminsky M, Fukada Y, Carter DA, Aguayo AJ, Vidal-Sanz M. Electrophysiologic responses in hamster superior colliculus evoked by regenerating retinal axons. Science 1989: 246: 255-257.

5 Schwab ME. Myelin-associated inhibitors of neurite growth and regeneration in the CNS. Trends in Neurosci 1990: 13:452-456.

6 Savio T, Schwab ME. Rat CNS white matter, but not gray matter, is nonpermissive for neuronal cell adhesion and fiber outgrowth. J Neurosci 1989: 9:1126-1133.

7 Schwab ME, Thoenen H. Dissociated neurons regenerate into sciatic but not optic nerve explants in culture irrespective of neurotrophic factors. J Neurosci 1985: 5:2414-2423.

8 Schwab ME, Caroni P. Oligodendrocytes and CNS myelin are nonpermissive substrates for neurite growth and fibroblast spreading in vitro. J Neurosci 1988: 8:2381-2393.

9 Bandtlow CE, Zachleder T, Schwab ME. Oligodendrocytes arrest neurite growth by contact inhibition. J Neurosci 1990: 10:3837-3848.

10 Caroni P, Schwab ME. Two membrane protein fractions from rat central myelin-with inhibitory properties for neurite growth and fibroblast spreading. J Cell Biol 1988: 106:1281-1288.

11 Caroni P, Schwab ME. Antibody against myelin-associated inhibitor of neurite growth neutralizes nonpermissive substrate properties of CNS white matter. Neuron 1988: 1:85-96. 
12 Gilmore SA. The effects of X-irradiation on the spinal cords of neonatal rats. II. Histological observations. J Neuropathol Exp Neurol 1963: 22:294-301.

13 Savio T, Schwab ME. Lesioned corticospinal tract axons regenerate in myelin-free rat spinal cord. Proc Natl Acad Sci USA 1990: 87:4130-4133.

14 Schnell L, Schwab ME. Axonal regeneration in the rat spinal cord produced by an antibody against myelin-associated neurite growth inhibitors. Nature 1990: 343:269-272.

15 Cadelli D, Schwab ME. Regeneration of lesioned septohippocampal acetylcholinesterase-positive axons is improved by antibodies against the myelin-associated neurite growth inhibitors NI-35/250. Europ J Neurosci 1991 (in press).

16 Kromer LF, Björklund A, Stenevi U. Regeneration of the septohippocampal pathways in adult rats is promoted by utilizing embryonic hippocampal implants as bridges. Brain Res 1981: 210:153-200.

17 Hagg T, Vahlsing HL, Manthorpe M, Varon S. Septohippocampal cholinergic axonal regeneration through peripheral nerve bridges: quantification and temporal development. Exp Neurol 1990: 109:153-163.

18 Barde YA. Trophic factors and neuronal survival. Neuron 1989: 2:1525-1534.

19 Kromer LF. Nerve growth factor treatment after brain injury prevents neuronal death. Science 1987: 235:214-216.

20 Tuszynski MH, Buzsaki G, Gage FH. Nerve growth factor infusions combined with fetal hippocampal grafts enhance reconstruction of the lesioned septohippocampal projection. Neurosci 1990: 36:33-44. 